\title{
Considerations around the SARS-CoV-2 Spike Protein with Particular Attention to COVID-19 Brain Infection and Neurological Symptoms
}

\author{
Kambiz Hassanzadeh, Helena Perez Pena, Jessica Dragotto, Lucia Buccarello, Federico Iorio, \\ Stefano Pieraccini, Giulio Sancini, and Marco Feligioni*
}

Cite This: ACS Chem. Neurosci. 2020, 11, 2361-2369

Read Online

\section{ACCESS |}

Џlll Metrics \& More

Article Recommendations

Supporting Information

ABSTRACT: Spike protein (S protein) is the virus "key" to infect cells and is able to strongly bind to the human angiotensin-converting enzyme2 (ACE2), as has been reported. In fact, Spike structure and function is known to be highly important for cell infection as well as for entering the brain. Growing evidence indicates that different types of coronaviruses not only affect the respiratory system, but they might also invade the central nervous system (CNS). However, very little evidence has been so far reported on the presence of COVID-19 in the brain, and the potential exploitation, by this virus, of the lung to brain axis to reach neurons has not been completely understood. In this Article, we assessed the SARS-CoV and SARS-CoV-2 Spike protein sequence, structure, and electrostatic potential using computational approaches. Our results showed that the $S$ proteins of SARS-CoV-2

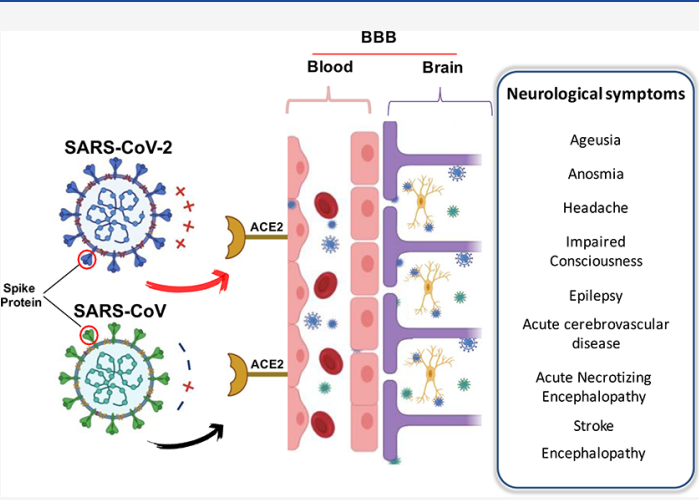
and SARS-CoV are highly similar, sharing a sequence identity of $77 \%$. In addition, we found that the SARS-CoV-2 S protein is slightly more positively charged than that of SARS-CoV since it contains four more positively charged residues and five less negatively charged residues which may lead to an increased affinity to bind to negatively charged regions of other molecules through nonspecific and specific interactions. Analysis the $S$ protein binding to the host ACE2 receptor showed a 30\% higher binding energy for SARS-CoV-2 than for the SARS-CoV S protein. These results might be useful for understanding the mechanism of cell entry, blood-brain barrier crossing, and clinical features related to the CNS infection by SARS-CoV-2.

KEYWORDS: ACE2, Brain, COVID-19, Spike Protein

\section{INTRODUCTION}

The crucial step in the viral infection is the process of viral entry into the host cells, and understanding this mechanism is important for exploring effective therapeutic agents for the treatment of viral infection. The endocytic pathway including endosomes and lysosomes and the autophagy process in viral entry has attracted considerable attention a therapeutic target in combating diseases caused by viruses in the past decade. ${ }^{1}$

The clathrin-dependent endocytotic/exocytotic pathway has been reported as the main pathway for some viruses enter host cells such as Hepatitis $C$ virus, Tick-borne encephalitis virus, and Zika virus which enter the astrocytes and induce neuroinfection by endocytosis, ${ }^{2,3}$ Whether SARS-CoV-2 infects the neuronal system by this mechanism has yet to be elucidated. Other types of coronavirus, such as swine hemagglutinating encephalomyelitis virus (HEV), employ endocytosis for trans-synaptic transfer. ${ }^{4}$

From a molecular point of view, computational modeling studies highlighted the huge similarity between SARS-CoV-2 with the original SARS-CoV especially in the three-dimensional (3D) structures of the receptor-binding domain of Spike proteins $(S)$. Several lines of evidence focused on Spike protein as a main tool of the virus to infect cells by strongly binding to the angiotensin converting enzyme 2 (ACE2)., ${ }^{5,6}$ The Spike protein is a homotrimer that protrudes from the viral membrane and contains, in each of its monomers, a receptor binding domain (RBD) through which this viral protein directly interacts with the ACE2 receptor located on the surface of many host cells. ${ }^{7-10}$ ACE2 is an enzyme attached to the outer surface (cell membranes) of cells in the lungs, arteries, heart, kidney, intestines, and brain. ${ }^{11}$ ACE2, which is expressed in the brain, mainly exists in the brain stem and in the regions involved in cardiovascular function and central regulation of blood pressure including the subfornical organ, nucleus of the tractus solitarius, paraventricular nucleus, and rostral ventrolateral medulla. ${ }^{12,13}$ In a previous study, Wrapp et

Received: June 15, 2020

Accepted: July 6, 2020

Published: July 6, 2020

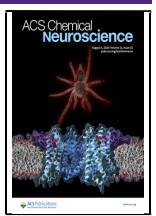




\begin{tabular}{|c|c|c|}
\hline SARS_COV_2 & 1 & MFVFLVLIPLVS----SQCVNLTTRTQLPPAYTNSFT--RGVYYPDKVFRSSVLHSTQDLFLPFFSNVTWFHAIHVSGTNGTKRFDNPVLPFNDGVYEA \\
\hline SARS_COV & 1 & MFIFLLFLTLTSGSDLDRCTTFDDVQ--APNXTQHTSSMRGVYYPDEIERSDTLYLTQDLELPFYSNVTGFHTIN-------HTEGNPVIPEKDGIYEA \\
\hline SARS_COV_2 & 94 & STEKSNIIRGWIFGTTLDSKTQSLLIVNNATNVVIKVCEFEFCNDPFLGVYYHKNNKSWMESEFRVYSSANNCTFEYVSQPFLMDLEGKQGNFKNLREF \\
\hline SARS_COV $^{-}$ & 91 & ATEKSNVVRGWVEGSTMNNKSQ SVIIINNSTNVVIRACNEELCDNPFFAV----SKPMGTQTHTMIFDNAFNCTEEYISDAESLDVSEKSENEKHLREE \\
\hline SARS_COV_2 & 193 & VFKNIDGYFKIYYSKHTPINLVRDLPQGFSALEPLVDLPIGINITRFQTLLALHRSYLTP̄GDSSSGWTTAGAAAYYVGYLQPRTFLLKYNENGTITDAVDC \\
\hline SARS_COV & 186 & VEKNKDGFLYVYKGYQPIDVVRDLPSGENTLKPIFKLPLGINITNERAILTA----FSPAQDI--WGTSAAAYFVGYLKPTTFMLKYDENGTITDAVDC \\
\hline SARS_COV_2 & 292 & ALDPLSETKCTLKSFTVEKGIYQTSNFRVQPTESIVRFPNITNLCPFGEVFNATRFASVYAWNRKRISNCVADYSVLYNSASFSTFKCYGVSPTKLNDI \\
\hline SARS_COV & 279 & SQNPLAELKCSVKSFEIDKGIYQTSNERVVRSGDVVRFPNITNLCPFGEVENATKEPSVYAWERKKISNCVADYSVLYNSTFESTEKCYGVSATKLNDL \\
\hline SARS_COV_2 & 391 & CFTNVYADSFVIRGDEVRQIAPGQTGKIADYNYKLPDDFTGCVIAWNSNNLDSKVGGNYNYLYRLFRKSNLKPFERDISTEIYQAGSTPCNGVEGFNCY \\
\hline SARS_COV $^{-}$ & 378 & CESNVYADSFVVKGDDVRQIAPGQTGVIADYNYKLPDDFMGCVLAWNTRNIDATSTGNYNYKYRYLRHGKLRPEERDISNVPFSPDGKPCTP-PALNCY \\
\hline SARS_COV_2 & 490 & FPLQSYGGEPTNGVGYQPYRVVVLSFELLHAPATVCGPKKSTNLVKNKCVNFNFNGLTGTGVLTESNKKFLPFQQFGRDIADTTDAVRDPQTLEILDIT \\
\hline SARS_COV & 476 & WPLNDYGEYTTTGIGYQPYRVVVLSFELLNAPATVCGPKLSTDLIKNQCVNFNENGLTGTGVLTPSSKREQPEQQEGRDVSDFTDSVRDPKTSEILDIS \\
\hline SARS_COV_2 2 & 589 & PCSFGGVSVITPGTNTSNQVAVLYQDVNCTEVPVAIHADQLTPTWRVYSTGSNVEQTRAGCLIGAEHVNNSYECDIPIGAGICASYQTQTNSPRRARSV \\
\hline SARS_COV & 575 & PCSFGGVSVITPGTNASSEVAVLYQDVNCTDVSTAIHADQLTPAWRIYSTGNNVEQTQAGCLIGAEHVDTSYECDIPIGAGICASYHTVS----LLRST \\
\hline SARS_COV_2 & 688 & ASQSIIAYTMSLGAENSVAYSNNSIAI PTNFTISVTTEILPVSMTKTSVDCTMY ICGDSTECSNLLLQYGSFCTQLNRALTGIAVEQDKNTQEVFAQVK \\
\hline SARS_COV & 670 & SQKSIVAYTMSLGADSSIAYSNNTIAIPTNESISITTEVMPVSMAKT SVDCNMYICGDSTECANLLLQYGSFCTQLNRALSGIAAEQDRNTREVEAQVK \\
\hline SARS_COV_2 & 787 & QIYKTPPIKDFGGFNFSQILPDPSKPSKRSFIEDLLFNKVTLADAGFIKQYGDCLGDIAARDLICAQKFNGLTVLPPLLTDEMIAQYTSALLAGTITSG \\
\hline $\mathrm{SARS}_{-}^{-} \mathrm{COV}^{-}$ & 769 & QMYKTPTLKYEGGFNESQILPDPLKPTKRSFIEDLLFNKVTLADAGFMKQYGECLGDINARDLICAQKFNGLTVLPPLLTDDMIAAYTAALVSGTATAG \\
\hline SARS_COV_2 & 886 & WTFGAGAALQIPFAMQMAYRFNGIGVTQNVLYENQKLIANQFNSAIGKIQDSLSSTASALGKLQDVVNQNAQALNTLVKQLSSNFGAISSVLNDILSRL \\
\hline SARS_COV & 868 & WTFGAGAALQIPEAMQMAYRFNGIGVTQNVLYENQKQIANQENKAISQIQESLTTTSTALGKLQDVVNQNAQALNTLVKQLSSNEGAISSVLNDILSRL \\
\hline SARS_COV_2 & 985 & DKVEAEVQIDRLITGRLQSLQTYVTQQLIRAAEIRASANLAATKMSECVLGQSKRVDFCGKGYHLMSFPQSAPHGVVFLHVTYVPAQEKNFTTAPAICH \\
\hline SARS_COV & 967 & DKVEAEVQIDRLITGRLQSLQTYVTQQLIRAAE IRASANLAATKMSECVLGQSKRVDFCGKGYHLMSEPQAAPHGVVELHVTYVPSQERNFTTAPAICH \\
\hline SARS_COV_2 & 1084 & ADGKAHFPREGVFVSNGTHWFVTQRNFYEPQIITTDNTFVSGNCDVVIGIVNNTVYDPLQPELDSFKEELDKYFKNHTSPDVDLGDISGINASVVNIQKE \\
\hline SARS_COV & 1 & 6EGKAYFPREGVEVFNGTSWEITQRNEFSPQIITTDNTFVSGNCDVVIGIINNTVYDPLQPELDSFKEELDKYFKNHTSPDVDLGDISGINASVVNIQKE \\
\hline SARS_COV_2 & 1183 & 3IDRLNEVAKNLNESLIDLQELGKYEQY IKWPWY IWLLGFIAGL IAIVMVTIMLCCMTSCCSCLKGCCSCGSCCKFDEDDS \\
\hline $\mathrm{SARS}_{-}^{-} \mathrm{COV}^{-}$ & 1 & 5 IDRLNEVAKNLNESLIDLQELGKYEQY IKWPWYVWLGEIAGL IAIVMVTILLCCMTSCCSCLKGACSCGSCCKFDEDDSEPVLKGVKLHYT \\
\hline
\end{tabular}

Figure 1. Sequence alignment of SARS-CoV-2 and SARS-CoV S protein. Conserved residues are labeled in dark green, the same residues are indicated in lighter green, and residues with similar properties are yellow.

al. reported that SARS-CoV-2 S protein exhibits higher binding affinity than that of SARS-CoV to the ACE2 receptor. ${ }^{14}$

SARS-CoV and SARS-CoV-2 share about $96 \%$ nucleotide sequence identity, suggesting that SARS-CoV-2 might have emerged from a bat SARS-like coronavirus. Therefore, in this study, we investigated the differences between the sequence, structure, and electrostatic potential of SARS-CoV-2 and SARS-CoV Spike proteins in both their open and closed conformations using computational approaches and discuss how these divergences may make this new virus highly infectious to human cells and organs with particular attention to brain infection and neurologic symptoms in patients with COVID-19.

In fact, although the most prevalent symptom that leads COVID-19 patients to the intensive care units is heavy respiratory complications, some patients also showed neurologic signs which have been described in three categories: central nervous system (CNS) symptoms or diseases (headache, dizziness, impaired consciousness, ataxia, acute cerebrovascular disease, and epilepsy), peripheral nervous system (PNS) symptoms (hypogeusia, hyposmia, and neuralgia), and musculoskeletal symptoms. ${ }^{15,16}$ Recently, more serious complications including acute encephalopathy ${ }^{17}$ and acute hemorrhagic necrotizing encephalopathy (ANE) ${ }^{18}$ have been reported in case report studies. ANE is a rare complication of viral infections such as influenza and has been related to a remarkable increase in intracranial cytokines, which leads to blood-brain barrier (BBB) breakdown. ${ }^{19}$
There is no evidence regarding the entry of SARS-CoV-2 to the brain to date in either animal or human studies. Indeed, several papers reported the presence of SARS-CoV in the central nervous system (CNS) and found in cerebrospinal fluid (CSF) like in the report in which the status epilepticus of a patient was associated with SARS $^{20}$ and others reports in which demyelinating brain pathology has been associated with coronaviruses infection. ${ }^{21}$ Moreover, some clinical studies performed on patients affected by SARS-CoV have identified the presence of virus particles in the brain, mainly localized in the neurons. ${ }^{22-24}$ Therefore, in this Article, we also discuss the possible pathological interaction between the brain and lungs, CNS infection, and relevant clinical futures in patients with COVID-19 based on our current knowledge.

\section{RESULTS AND DISCUSSION}

The results of sequence alignment show that the sequences of the $\mathrm{S}$ proteins of SARS-CoV-2 and SARS-CoV are highly similar, sharing a sequence identity of $77 \%$. Nonetheless, some divergences can be observed in the sequence (Figure 1 and Supplementary Data). These divergences have been examined in a previous study by Jaimes et al., ${ }^{25}$ who reported the $3 \mathrm{D}$ structures of the proteins. ${ }^{25}$ Moreover, Baig et al. suggested that these differences may be related to the higher binding affinity of SARS-CoV-2 S protein to the host ACE2 receptor. ${ }^{26}$

More recently, Robson indicated that all human SARS coronaviruses (and indeed the Spike proteins of many other related coronaviruses) appear to be similar in general 
conformation, and the variations observed in experimental structures probably have more to do with crystallization or other preparation methods. ${ }^{27}$

Our findings reveal that the SARS-CoV-2 $S$ protein is slightly more positively charged than that of SARS-CoV since it contains four more positively charged residues and five less negatively charged residues (Table 1 ). Even if the difference in

Table 1. Number of Each Residue Present in the $S$ Protein of SARS-CoV-2 and SARS-CoV ${ }^{a}$

\begin{tabular}{ccc} 
residue & SARS-CoV-2 & SARS-CoV \\
A & 79 & 84 \\
R & 42 & 39 \\
N & 88 & 81 \\
D & 62 & 73 \\
C & 40 & 39 \\
Q & 62 & 55 \\
E & 48 & 42 \\
G & 82 & 79 \\
H & 17 & 15 \\
I & 76 & 78 \\
L & 108 & 99 \\
K & 61 & 60 \\
M & 14 & 20 \\
F & 77 & 83 \\
P & 58 & 57 \\
S & 99 & 96 \\
T & 97 & 99 \\
W & 12 & 11 \\
Y & 54 & 54 \\
V & 97 & 91 \\
\hline
\end{tabular}

${ }^{a}$ The different residues are represented by a one letter code. Positively charged residues are indicated in italic, and negatively charged residues are indicated in bold.

charge between SARS-CoV-2 and SARS-CoV S proteins is rather small, this effect can be amplified by the high number of $S$ proteins that are present on a virus particle. This difference in charge between SARS-CoV-2 and SARS-CoV S proteins can have a significant impact in cell adhesion and crossing of the $\mathrm{BBB}^{28,29}$ which will be discussed more in detail later in this Article.

A two-step process takes place when the $S$ proteins interact with other proteins, such as when the $S$ protein binds to the human ACE2 receptor, to establish a final protein-protein association. (1) The first step is dominated by electrostatic forces that lead $t$ the formation of an ensemble of transient and nonspecific encounter complexes. ${ }^{30}$ In this step, the $S$ protein would be found in the closed conformation. (2) A structural rearrangement takes place in the protein, and the three $S$ protein RBDs open up to expose their binding interface to form a well-defined complex, which is stabilized not only by electrostatic forces, but also by polar (salt-bridge and hydrogen bond) and nonpolar interactions ( $\pi$-stack, $\pi$-anion, and shortrange hydrophobic interactions). ${ }^{7,30}$ Taking this into account, the electrostatic potential of both SARS-CoV-2 and SARS-CoV $S$ protein surfaces, both in the open and closed conformations, has been calculated in this study (see Figure 2), also focusing in their RBDs, in order to analyze the differences in the ability of SARS-CoV-2 and SARS-CoV to bind to other molecules within the human body according to their electrostatic properties and, thus, their capacity to enter human cells.
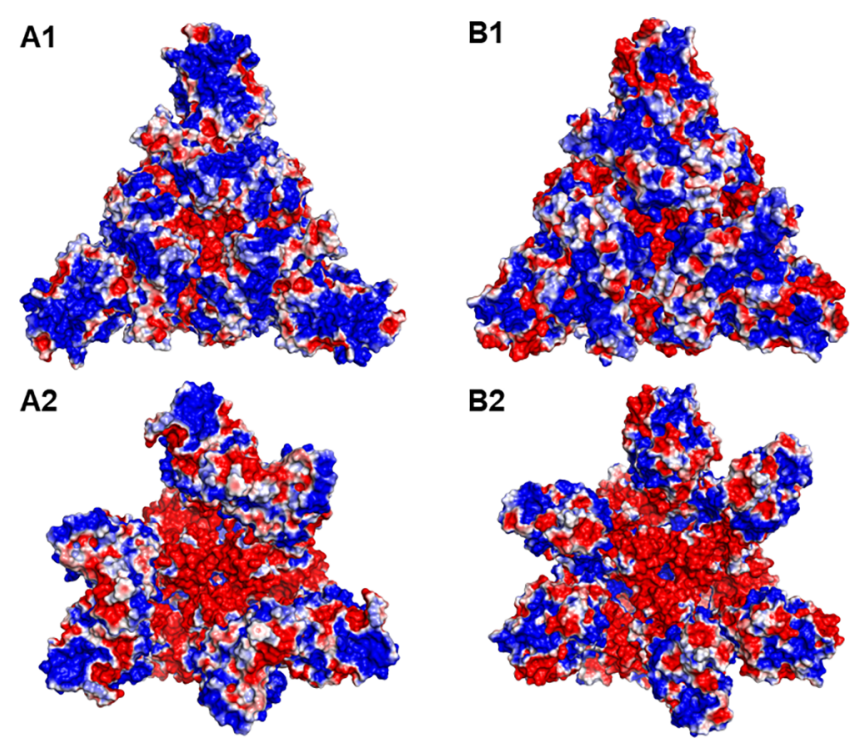

Figure 2. Electrostatic potential of Spike protein in SARS-CoV-2 vs SARS-CoV. Electrostatic potential of (A) SARS-CoV-2 and (B) SARS-CoV S protein in the (1) closed and (2) open conformations mapped onto their molecular surface. This region represents the top side of the protein where the RBD is located and thus the opposite side to the one that is attached to the surface of the virus. The negative electrostatic potential is shown in red, the neutral in white, and the positive in blue. Values range from $-k T / e$ (red) to $+k T / e$ (blue).

Several structures of $S$ proteins could be found in the Protein Data Bank (PDB), but unresolved segments were present in all of them. In order to calculate and map a protein electrostatic potential, a complete structure is needed; therefore, complete 3D structures of SARS-CoV-2 and SARS-CoV protein $S$, in both the open and closed conformation, were modeled using homology modeling techniques. Having modeled SARS-CoV-2 and SARS-CoV S protein structures, both structures in the close state conformation were superimposed with a $1.236 \AA$ root-meansquare deviation (RMSD) over 427 aligned $\mathrm{C} \alpha$ positions. In this way, the structure of both proteins was compared, showing a high structure similarity. Afterward, macromolecular electrostatic calculations of the models were performed. In other studies, differences in the RBD:ACE2 interfaces between the SARS-CoV-2 and SARS-CoV S protein at a structural level were described in detail and have been linked to SARS-CoV-2 higher binding affinity. Herein, these interfaces have been analyzed at the electrostatic potential level (see Figures 3 and 4).

Recently, in a report published in Nature, Lan et al., identified residues in the SARS-CoV-2 RBD that are essential for ACE2 binding, the majority of which either are highly conserved or share similar side chain properties with those in the SARS-CoV RBD. They believe that the similarity in structure and sequence strongly indicates convergent evolution between the SARS-CoV-2 and SARS-CoV RBDs for improved binding to ACE2. ${ }^{31}$

In Figure 2, the electrostatic potentials of SARS-CoV-2 and SARS-CoV S protein (top side) are compared, showing that the SARS-CoV-2 $S$ protein surface exhibits a more positive electrostatic potential than that of SARS-CoV. This same electrostatic potential difference can also be seen in the binding interface of their RBDs (Figure 3). Thus, despite presenting a 

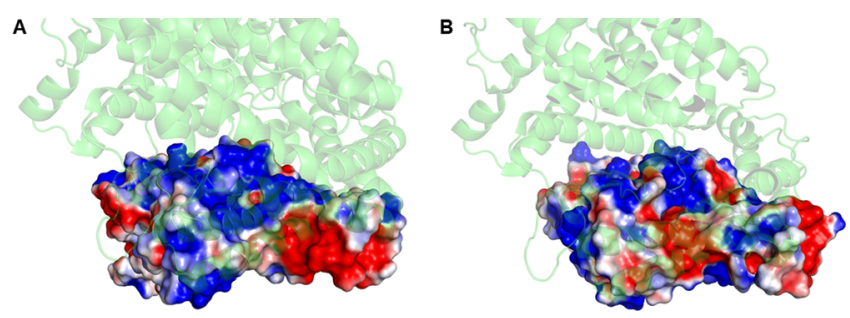

Figure 3. Electrostatic potential of (A) SARS-CoV-2 (PDB ID GLZG) and (B) SARS-CoV (PDB ID 6ACJ, A) S protein RBD section mapped onto its molecular surface when in complex with human ACE2 receptor (transparent green). The negative electrostatic potential is shown in red, the neutral in white, and the positive in blue. Values range from $-k T / e$ (red) to $+k T / e$ (blue).
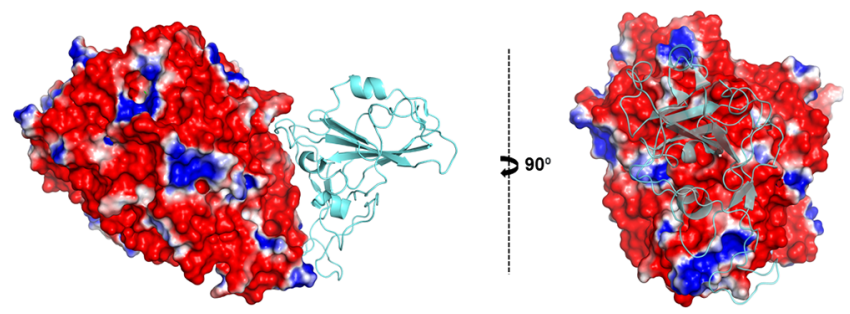

Figure 4. Electrostatic potential of human ACE2 receptor. Electrostatic potential of human ACE2 receptor mapped onto its molecular surface when in complex with SARS-CoV-2 (cyan) (PDB ID GLZG) shown from different perspectives. The negative electrostatic potential is shown in red, the neutral in white, and the positive in blue. Values range from $-k T / e$ (red) to $+k T / e$ (blue).

high sequence and structural similarity, SARS-CoV-2 and SARS-CoV S proteins have different electrostatic properties. This difference can have an effect on the capacity of the virus to adhere to other molecules. On the other side, human ACE2 binding interface tends to have a predominantly negative electrostatic potential (Figure 4) and, therefore, will interact more strongly with the SARS-CoV-2 $\mathrm{S}$ protein both in the open and close conformations.

A comparison of SARS-CoV-2 and SARS-CoV $S$ protein sequences, $3 \mathrm{D}$ structures, and electrostatic potentials reveals that both proteins have a conserved sequence and structural features but different electrostatic characteristics in both their external surface and their host-interaction interfaces. As previously described, the SARS-CoV-2 $\mathrm{S}$ protein is slightly more positively charged in these regions than SARS-CoV is, which will lead to an increased affinity to bind to negatively charged regions of other molecules through nonspecific and specific interactions.

Moreover, some differences in the amino acidic content of the $S$ protein in the RBD-ACE2 interface can lead to the establishment of more specific interactions with the host receptors. Hence, SARS-CoV-2 is more likely to establish interactions with different targets across the human body than SARS-CoV both through nonspecific and specific interactions. All this, ultimately, can increase the capacity of SARS-CoV-2 to enter human cells and bind to the negative charge barriers such as the $\mathrm{BBB}^{32}$ with respect to SARS-CoV.

In the last months, $S$ protein structure and electrostatic properties have been the subject of much investigation. Previous computer-based experiments have also noted that the SARS-CoV-2 RBD exhibits a more positive electrostatic potential than the SARS-CoV RBD does ${ }^{31,33-35}$ and that the electrostatic potential has a particularly important role in the high infection rate of SARS-CoV-2. In agreement with our results, it has previously been observed that SARS-CoV-2 binds with a higher affinity to the human ACE2 receptor than SARS$\mathrm{CoV}$ does. ${ }^{33}$ This was also attributed to the enhanced electrostatic interactions between SARS-CoV-2 and ACE2 due to the SARS-CoV-2 RBD having greater electrostatic complementarity with the binding domain of ACE2 than the SARS-CoV RBD. ${ }^{33}$ In particular, it has been reported that the increased positive electrostatic potential of the SARS-CoV-2 binding surface is mainly due to an essential mutation of the hydrophobic residue Val404, present in SARS-CoV, to the positively charged residue Lys417 in SARS-CoV-2. ${ }^{31,34}$ Amin et al. also identified a complementary negative electrostatic potential on the surface of the binding site of ACE2. ${ }^{33}$

Taking advantage of our previous experience dealing with nanoparticles (NPs) specifically tailored to cross the BBB and target the brain tissue, we can speculate the potential strategies of SAR-CoV-2 to enter into the brain. Indeed, the dimension and the surface properties of the SAR-CoV-2 are similar, in terms of adhesion and cell membrane crossing abilities, to those shown by the nanoparticles specifically designed for BBB crossing. ${ }^{29,36}$ So the parallelism between SAR-CoV-2 and the strategies adopted to let nanoparticles cross the BBB can be useful to hypothesize the ways used by the virus to enter into the brain. Therefore, an increase of the number of the positive amino acids of the SAR-CoV-2 envelope might increase in a significant manner the adhesion properties of SAR-CoV-2 crossing the $\mathrm{BBB}$ and entering the brain.

In order to quantify the difference in the binding affinity of the two complexes (SARS-CoV-2:ACE2 and SARS-Co$\mathrm{V}: A C E 2)$, their binding free energy was calculated. The results showed that SARS-CoV-2 S protein binds to the host ACE2 receptor with a $30 \%$ higher binding energy than the SARS$\mathrm{CoV} S$ protein does. It has also been observed that the electrostatic contribution to the total binding free energy is the dominant term in the SARS-CoV-2:ACE2 interaction. Hence, this data supports the qualitative analysis of the electrostatic potential of the structures presented above and the quantitative data shown in previous studies.

According to the bioinformatics data regarding the possible interaction between the virus Spike protein and ACE2 protein, it is suggested that it is probable for SARS-CoV-2 to adhere with higher efficiency to the cells through nonspecific interactions which have a major impact on cell adhesion ${ }^{28}$ due to (1) SARS-CoV-2 electrostatic properties and (2) binding with higher affinity to the host ACE2 receptor through specific interactions. In fact, our findings revealed that the Spike protein of SARS-CoV-2 binds to the host ACE2 receptor with a significantly higher binding energy than the SARS-CoV $S$ protein does, indicating that the electrostatic contribution to the total binding free energy is the dominant term in the SARS-CoV-2:ACE2 interaction.

As previously described, Spike protein and ACE2 represent the key, but not the exclusive, site of entry of the virus into the cell; thus, non-ACE2 pathways for virus infection of neural cells cannot be excluded. ${ }^{37}$ Whether COVID-19 infects neurons and astroglial cells and enters astrocytes by endocytosis remains to be studied. Overall, considering the computational assays that have been performed in this study, we suggest that the Spike protein dependent pathway is thought to be more important than clathrin-dependent endocytosis for cell entry and BBB crossing. Therefore, the Spike dependent pathway should be taken into account in 
therapeutic strategies for specific antibodies or vaccine production research.

Regardless of how the virus enters the brain, there are some CNS complications in patients with COVID-19 that should be taken into consideration. The presence of the virus in the brain stem may affect chemosensing neural cells related to respiration as well as respiratory center neurons, thus damaging the lung ventilatory function. ${ }^{37}$ It has been shown that SARS$\mathrm{CoV}$ downregulates ACE2 protein expression in a replication dependent manner. ${ }^{38}$ Supporting these findings, it has been revealed that SARS-CoV infections and the Spike protein of SARS-CoV reduced ACE2 expression and the injection of SARS-CoV Spike into mice worsened acute lung failure in vivo, which was attenuated by blocking the renin-angiotensin pathway. $^{39}$

Considering the high similarity of SARS-CoV and SARCoV-2, and higher binding energy of SAR-CoV-2 than the SARS-CoV S protein to bind ACE2, it has been hypothesized that SARS-CoV-2 also can downregulate ACE2 in different organs including the brain. ${ }^{40,41}$ This downregulation might be a part of this complicated story; inhibition of ACE2 activity reduces the sensitivity of the baroreceptor reflex control of the heart rate as well as increases sympathetic tone, eventually resulting in blood pressure elevation and cardiac dysfunction. On the other hand, an increase of inflammatory cytokines during lung injury, hypoxemia, and elevation of sympathetic tone through ACE2 downregulation leads to CNS hyperactivation which might play a crucial role in the etiopathogenesis of neurogenic pulmonary edema (NPE), ${ }^{42}$ a lifethreatening complication following a neurologic insult, ${ }^{43}$ and finally leading to deterioration with the respiratory and cardiovascular complications in these patients (see Figure 5).

Supporting the idea of brain infection, more recently, in a case report, one patient with no past medical history showed frequent seizures probably due to COVID-19 infection. ${ }^{44}$ Several mechanisms for the etiology of seizure have been taken into consideration, including the direct infiltration of brain tissue, production of toxins by the virus, or increase of inflammatory cytokines by the brain. ${ }^{45}$ Recently, It has been reported that COVID-19 initiates the inflammatory cascade and, as a result, releases inflammatory cytokines ${ }^{46}$ which is called cytokine storm syndrome. ${ }^{47}$ Consecutively, these cytokines can drive neuronal hyperexcitability via activation of glutamate receptors and play a role in the development of acute seizures. ${ }^{4-50}$

In addition, in a case report study, a case of self-limited encephalitic associated with SARS-CoV-2 was presented. The authors suggested that, with the clearance of the virus and the use of mannitol, CSF pressure might gradually decrease and the patient's consciousness will improve. ${ }^{51}$

In a recent study, neurologic features in severe COVID-19 patients who were admitted to the hospital have been reported. Magnetic resonance imaging (MRI) of the brain was performed in 13 patients in this evaluation. Although these patients did not have focal signs that suggested stroke, they underwent MRI because of unexplained encephalopathic features. Two of the 13 patients who underwent brain MRI showed single acute ischemic strokes. The authors concluded that their data were not enough to recognize which of these features were due to critical illness-related encephalopathy, cytokines, or the effect or withdrawal of medication and which features were directly due to SARS-CoV-2 infection.

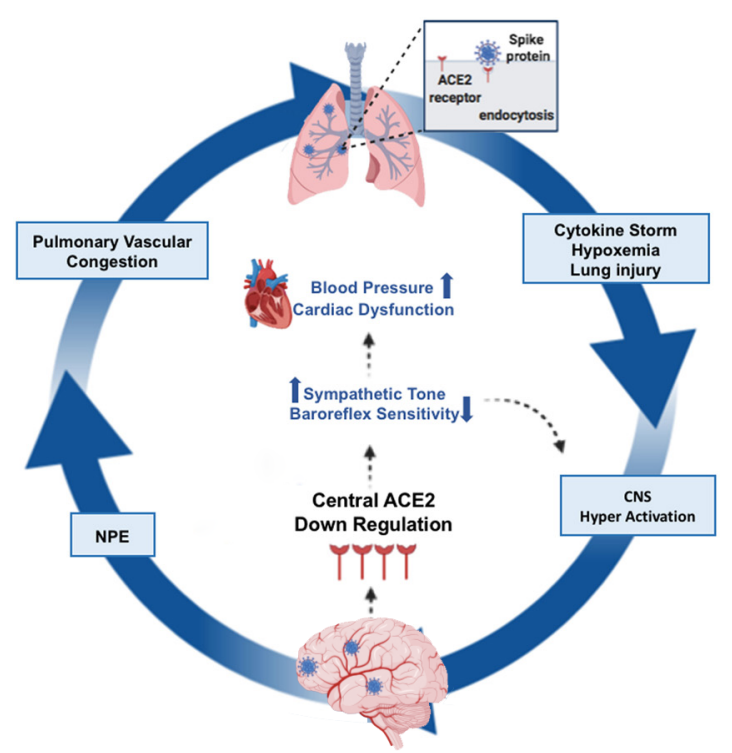

Figure 5. Brain and lung crosstalk during COVID-19 infection. SARCoV-2 employs ACE2 as the receptor for viral cell entry and induction of lung injury through increasing the immune system cytokines. It can downregulate the central ACE2 protein expression; inhibition of ACE2 activity reduces the sensitivity of the baroreceptor reflex control of the heart rate as well as increases sympathetic tone which eventually results in the blood pressure elevation and cardiac dysfunction. In addition, concerning the neuroprotective property of ACE2, its downregulation may disturb the balance of neurotoxicity/ neuroprotection inside the brain. Increase of inflammatory cytokines during lung injury, hypoxemia, and elevation of sympathetic tone through ACE2 downregulation leads to CNS hyperactivation which might play a crucial role in the etiopathogenesis of neurogenic pulmonary edema which may play a role in COVID-19 pulmonary complications in patients. ACE2, angiotensin-converting enzyme 2; NPE, neurogenic pulmonary edema; NP, neuroprotection; NT, neurotoxicity.

Postviral anosmia, which is also named olfactory dysfunction, ${ }^{52,53}$ and ageusia ${ }^{54}$ are other neurologic symptoms that have been reported in patients with COVID-19. More recently, in a cross-sectional study in Iran of 10,069 cases, the coincidence of COVID-19 epidemic and olfactory dysfunction has been reported. ${ }^{55}$ In this context, recently, Lechien et al. reported that olfactory and gustatory dysfunctions are prevalent in patients with mild-to-moderate COVID-19 infection. ${ }^{56}$ Some mechanisms have been raised to explain this association including (1) injury at the level of the neuroepithelium of olfactory receptor cells in the nasal roof or in the central olfactory processing system, ${ }^{55}$ (2) damage of the central olfactory routes and other regions of the brain, ${ }^{57-59}$ and (3) inflammation or possible damage to the nasal epithelium cells that are required for normal olfactory function. ${ }^{60}$ Therefore, both epithelial damage and CNS involvement have been reported as the possible causes; however, the exact pathophysiology remains yet to be elucidated. ${ }^{53,61}$

In accordance with the neurotrophic mechanism proposed by Baig et al., ${ }^{10}$ which hypothesizes SAR-CoV-2 brain access via the transcribrial route, as documented for other CNS targeting pathogens, we suppose a possible entry of the virus from the olfactory bulb and, exploiting the blood microcirculation, SAR-CoV-2 may have access to the cerebral 
circulation and interact with ACE2 receptors expressed on neuronal cells.

\section{CONCLUSION}

Considering the neurological manifestations of patients with COVID-19 and in light of the bioinformatics findings of this study indicating more positive charged Spike protein structure and higher binding free energy of the SARS-CoV-2:ACE2 interaction, it is expected that SAR-CoV-2 possesses higher efficiency than SARS-CoV to enter the cells and reach the brain. This neuroinvasive characteristic should be taken into account in basic and clinical research as well as prioritization and individualization of therapeutic approaches.

\section{METHODS}

Spike Protein Sequence Alignment and Analysis. The sequence alignment of the $S$ protein of SARS-CoV-2 (UniProt ID PODTC2) and SARS-CoV (UniProt ID P59594) was conducted in the Web server BLASTp ${ }^{62}$ using the Needleman-Wüncsh algorithm with the default substitution matrix (BLOSUM62) ${ }^{63}$ (see Figure 1). For illustrative purposes, the resulting sequence alignment was downloaded as a text file from BLASTp and converted into an ALI format file in order to visualize and produce the sequence alignment images on the Molsoft Browser 3.9. ${ }^{64,65}$ In order to analyze the divergence in the amino acidic content of the $S$ protein from SARSCoV-2 and SARS-CoV, the number of each residue present in each protein sequence was counted using the "str_count" function in RStudio 3.6.3 (Table 1). ${ }^{66}$

Homology Modeling and Structure Comparison. Homology models of the complete 3D structures of SARS-CoV-2 and SARS-CoV $S$ protein, both in the open and closed conformation, were built in the MODELER $9.23^{67}$ program by using a sequence alignment extracted from BLASTp ${ }^{62}$ and template structures obtained from the $\mathrm{PDB}^{68}$ (Supporting Information). Homology models of SARS-CoV-2 and SARS-CoV S proteins in the closed conformation were superimposed using the structure comparison tool Match Maker in the software UCSF Chimera 1.14. ${ }^{69}$

Calculation of the Electrostatic Potential. Electrostatic potentials of the homology models were calculated using the program Adaptive Poisson-Boltzmann Solver (APBS) ${ }^{70}$ and were displayed in PyMol 2.3.4 as a color-coded electrostatic potential molecular surface (Solvent-Excluded Surfaces (SESs)) by using the APBS 1.5 plugin $^{72}$ (see Figure 2).

Binding Free Energy Calculation. In order to quantify the difference in the binding affinity of complexes SARS-CoV-2:ACE2 and SARS-CoV:ACE2, their binding free energy was calculated using the Molecular Mechanics-Poisson-Boltzmann Surface Area (MMPBSA) approach ${ }^{73}$ implemented in the GROMACS-5.0.7 tool g_mmpbsa. ${ }^{74}$ MM-PBSA is a fully atomistic method for the calculation of binding free energies that combines a molecular mechanics description of the protein complex with a continuous solvent model. It is widely used to evaluate interaction energies between proteins and biomolecules in general. ${ }^{75}$

The structures of the SARS-CoV-2 S protein in complex with the ACE2 receptor (PDB ID 6LZG) and the SARS-CoV S protein in complex with the ACE2 receptor (PDB ID 6ACG) were subjected to geometry optimization in GROMACS-5.0.7 ${ }^{76}$ prior to calculations. A relative dielectric constant $\varepsilon=80$ was used to model the water solvent, while $\varepsilon=2$ was used for the protein in the solution of the Poisson-Boltzmann equation.

\section{ASSOCIATED CONTENT}

\section{sI Supporting Information}

The Supporting Information is available free of charge at https://pubs.acs.org/doi/10.1021/acschemneuro.0c00373.

Table of detailed sequence alignments used for homology modeling (PDF)

\section{AUTHOR INFORMATION}

\section{Corresponding Author}

Marco Feligioni - Laboratory of Neuronal Cell Signaling, EBRI Rita Levi-Montalcini Foundation, Rome 00161, Italy; Department of Neurorehabilitation Sciences, Casa di Cura del Policlinico, Milan 20144, Italy; $\odot$ orcid.org/0000-0001-70709662; Phone: +39 0649255 255; Email: m.feligioni@ ebri.it; Fax: +390649255255

\section{Authors}

Kambiz Hassanzadeh - Laboratory of Neuronal Cell Signaling, EBRI Rita Levi-Montalcini Foundation, Rome 00161, Italy; Cellular and Molecular Research Center, Research Institute for Health Development, Kurdistan University of Medical Sciences, Sanandaj 66177-13446, Iran

Helena Perez Pena - Department of Chemistry and National Inter-University Consortium for Materials Science and Technology-INSTM-UdR Milano, University of Milan, Milan 20133, Italy

Jessica Dragotto - Laboratory of Neuronal Cell Signaling, EBRI Rita Levi-Montalcini Foundation, Rome 00161, Italy

Lucia Buccarello - Laboratory of Neuronal Cell Signaling, EBRI Rita Levi-Montalcini Foundation, Rome 00161, Italy

Federico Iorio - Laboratory of Neuronal Cell Signaling, EBRI Rita Levi-Montalcini Foundation, Rome 00161, Italy

Stefano Pieraccini - Department of Chemistry and National Inter-University Consortium for Materials Science and Technology-INSTM-UdR Milano, University of Milan, Milan 20133, Italy; Institute of Science and Chemical Technology "Giulio Natta", Milan 20133, Italy; ○ orcid.org/0000-00027672-0720

Giulio Sancini - Human Physiology Lab, School of Medicine and Surgery, University of Milano-Bicocca, 20900 Monza, Italy

Complete contact information is available at:

https://pubs.acs.org/10.1021/acschemneuro.0c00373

\section{Author Contributions}

Co-last author: G.S. K.H.: Design, investigation, and writing of the original draft. H.P.P.: Methodology, investigation, and figure and table preparation. J.D.: Figure preparation and writing of the original draft. L.B.: Revisiion of the manuscript critically for important intellectual content. F.I.: Performed the literature search. S.P.: Design and methodology. G.S.: Cosupervision of project. M.F.: Conceiving the idea, design of the study, and supervision of project, funds. The manuscript was reviewed by all authors.

\section{Notes}

The authors declare no competing financial interest.

\section{REFERENCES}

(1) Yang, N., and Shen, H.-M. (2020) Targeting the Endocytic Pathway and Autophagy Process as a Novel Therapeutic Strategy in COVID-19. Int. J. Biol. Sci. 16 (10), 1724-1731.

(2) Jorgačevski, J., Korva, M., Potokar, M., Lisjak, M., Avšič-Županc, T., and Zorec, R. (2019) ZIKV Strains Differentially Affect Survival of Human Fetal Astrocytes versus Neurons and Traffic of ZIKV-Laden Endocytotic Compartments. Sci. Rep. 9 (1), 1-14.

(3) Zorec, R., Županc, T. A., and Verkhratsky, A. (2019) Astrogliopathology in the Infectious Insults of the Brain. Neurosci. Lett. 689, 56-62.

(4) Li, Y.-C., Bai, W.-Z., Hirano, N., Hayashida, T., Taniguchi, T., Sugita, Y., Tohyama, K., and Hashikawa, T. (2013) Neurotropic Virus Tracing Suggests a Membranous-Coating-Mediated Mechanism for Transsynaptic Communication. J. Comp. Neurol. 521 (1), 203-212. 
(5) Li, F., Li, W., Farzan, M., and Harrison, S. C. (2005) Structure of SARS Coronavirus Spike Receptor-Binding Domain Complexed with Receptor. Science 309 (5742), 1864-1868.

(6) Xu, X., Chen, P., Wang, J., Feng, J., Zhou, H., Li, X., Zhong, W., and Hao, P. (2020) Evolution of the Novel Coronavirus from the Ongoing Wuhan Outbreak and Modeling of Its Spike Protein for Risk of Human Transmission. Sci. China: Life Sci. 63 (3), 457-460.

(7) Xie, L., Sun, C., Luo, C., Zhang, Y., Zhang, J., Yang, J., Chen, L., Yang, J., and Li, J. (2020) SARS-CoV-2 and SARS-CoV Spike-RBD Structure and Receptor Binding Comparison and Potential Implications on Neutralizing Antibody and Vaccine Development. bioRxiv, February 20, 2020, ver. 1, DOI: 10.1101/2020.02.16.951723.

(8) Yan, R., Zhang, Y., Li, Y., Xia, L., Guo, Y., and Zhou, Q. (2020) Structural Basis for the Recognition of SARS-CoV-2 by Full-Length Human ACE2. Science 367 (6485), 1444-1448.

(9) Ortega, J. T., Serrano, M. L., Pujol, F. H., and Rangel, H. R. (2020) Role of Changes in Sars-Cov-2 Spike Protein in the Interaction With the Human Ace2 Receptor: An in Silico Analysis. EXCLI J. 19, 410-417.

(10) Baig, A. M., Khaleeq, A., Ali, U., and Syeda, H. (2020) Evidence of the COVID-19 Virus Targeting the CNS: Tissue Distribution, Host-Virus Interaction, and Proposed Neurotropic Mechanisms. ACS Chem. Neurosci. 11 (7), 995-998.

(11) Doobay, M. F., Talman, L. S., Obr, T. D., Tian, X., Davisson, R. L., and Lazartigues, E. (2007) Differential Expression of Neuronal ACE2 in Transgenic Mice with Overexpression of the Brain ReninAngiotensin System. Am. J. Physiol. Regul. Integr. Comp. Physiol. 292 (1), R373-381.

(12) Gowrisankar, Y. V., and Clark, M. A. (2016) Angiotensin II Regulation of Angiotensin-Converting Enzymes in Spontaneously Hypertensive Rat Primary Astrocyte Cultures. J. Neurochem. 138 (1), 74-85.

(13) Xia, H., and Lazartigues, E. (2010) Angiotensin-Converting Enzyme 2: Central Regulator for Cardiovascular Function. Curr. Hypertens. Rep. 12 (3), 170-175.

(14) Wrapp, D., Wang, N., Corbett, K. S., Goldsmith, J. A., Hsieh, C. L., Abiona, O., Graham, B. S., and McLellan, J. S. (2020) Cryo-EM Structure of the 2019-NCoV Spike in the Prefusion Conformation. Science 367 (6483), 1260-1263.

(15) Li, Y.-C., Bai, W.-Z., and Hashikawa, T. (2020) The Neuroinvasive Potential of SARS-CoV2May Play a Role in the Respiratory Failure of COVID-19 Patients. J. Med. Virol. 92, 552.

(16) Mao, L., Wang, M., Chen, S., He, Q., Chang, J., Hong, C., Zhou, Y., Wang, D., Li, Y., Jin, H., and Hu, B. (2020) Neurological Manifestations of Hospitalized Patients with COVID-19 in Wuhan, China: A Retrospective Case Series Study. medRxiv, February 25, 2020, ver. 1, DOI: 10.1101/2020.02.22.20026500.

(17) Filatov, A., Sharma, P., Hindi, F., and Espinosa, P. S. (2020) Neurological Complications of Coronavirus Disease (COVID-19): Encephalopathy. Cureus J. Med. Sci. 12 (3), e7352.

(18) Poyiadji, N., Shahin, G., Noujaim, D., Stone, M., Patel, S., and Griffith, B. (2020) COVID-19-Associated Acute Hemorrhagic Necrotizing Encephalopathy: CT and MRI Features. Radiology, 201187.

(19) Rossi, A. (2008) Imaging of Acute Disseminated Encephalomyelitis. Neuroimaging Clin. N. Am. 18 (1), 149-161.

(20) Hung, E. C. W., Chim, S. S. C., Chan, P. K. S., Tong, Y. K., Ng, E. K. O., Chiu, R. W. K., Leung, C.-B., Sung, J. J. Y., Tam, J. S., and Lo, Y. M. D. (2003) Detection of SARS Coronavirus RNA in the Cerebrospinal Fluid of a Patient with Severe Acute Respiratory Syndrome. Clin. Chem. 49 (12), 2108-2109.

(21) He, L., Ding, Y., Che, X., Zhang, Q., Huang, Z., Wang, H., Shen, H., Li, Z., Cai, J., Zhang, J., Geng, J., Li, X., Zhang, W., Han, H., Kang, W., Yang, L., and Lu, Y. (2003) [Expression of the monoclonal antibody against nucleocapsid antigen of SARS-associated coronavirus in autopsy tissues from SARS patients]. 1 Jun Yi Xue Xue Bao Acad. J. First Med. Coll. PLA 23 (11), 1128-1130.

(22) Ding, Y., He, L., Zhang, Q., Huang, Z., Che, X., Hou, J., Wang, H., Shen, H., Qiu, L., Li, Z., Geng, J., Cai, J., Han, H., Li, X., Kang, W.,
Weng, D., Liang, P., and Jiang, S. (2004) Organ Distribution of Severe Acute Respiratory Syndrome (SARS) Associated Coronavirus (SARS$\mathrm{CoV}$ ) in SARS Patients: Implications for Pathogenesis and Virus Transmission Pathways. J. Pathol. 203 (2), 622-630.

(23) Gu, J., Gong, E., Zhang, B., Zheng, J., Gao, Z., Zhong, Y., Zou, W., Zhan, J., Wang, S., Xie, Z., Zhuang, H., Wu, B., Zhong, H., Shao, H., Fang, W., Gao, D., Pei, F., Li, X., He, Z., Xu, D., Shi, X., Anderson, V. M., and Leong, A. S.-Y. (2005) Multiple Organ Infection and the Pathogenesis of SARS. J. Exp. Med. 202 (3), 415-424.

(24) Xu, J., Zhong, S., Liu, J., Li, L., Li, Y., Wu, X., Li, Z., Deng, P., Zhang, J., Zhong, N., Ding, Y., and Jiang, Y. (2005) Detection of Severe Acute Respiratory Syndrome Coronavirus in the Brain: Potential Role of the Chemokine Mig in Pathogenesis. Clin. Infect. Dis. 41 (8), 1089-1096.

(25) Jaimes, J. A., Andre, N. M., Chappie, J. S., Millet, J. K., and Whittaker, G. R. (2020) Structural Modeling of 2019-Novel Coronavirus (NCoV) Spike Protein Reveals a ProteolyticallySensitive Activation Loop as a Distinguishing Feature Compared to SARS-CoV and Related SARS-like Coronaviruses. J. Mol. Biol. 432 (10), 3309-3325.

(26) Baig, A. M., Khaleeq, A., Ali, U., and Syeda, H. (2020) Evidence of the COVID-19 Virus Targeting the CNS: Tissue Distribution, Host-Virus Interaction, and Proposed Neurotropic Mechanisms. ACS Chem. Neurosci. 11, 995.

(27) Robson, B. (2020) COVID-19 Coronavirus Spike Protein Analysis for Synthetic Vaccines, a Peptidomimetic Antagonist, and Therapeutic Drugs, and Analysis of a Proposed Achilles' Heel Conserved Region to Minimize Probability of Escape Mutations and Drug Resistance. Comput. Biol. Med. 121, 103749.

(28) Bongrand, P. (1998) Specific and Nonspecific Interactions in Cell Biology. J. Dispersion Sci. Technol. 19 (6-7), 963-978.

(29) Sancini, G., Gregori, M., Salvati, E., Cambianica, I., Re, F., Ornaghi, F., Canovi, M., Fracasso, C., Cagnotto, A., Colombo, M., Zona, C., Gobbi, M., Salmona, M., La Ferla, B., Nicotra, F., and Masserini, M. (2013) Functionalization with TAT-Peptide Enhances Blood-Brain Barrier Crossing in Vitro of Nanoliposomes Carrying a Curcumin-Derivative to Bind Amyloid- $\beta$ Peptide. J. Nanomed. Nanotechnol. 4 (3), 1000171.

(30) Johansson, H., Jensen, M. R., Gesmar, H., Meier, S., Vinther, J. M., Keeler, C., Hodsdon, M. E., and Led, J. J. (2014) Specific and Nonspecific Interactions in Ultraweak Protein-Protein Associations Revealed by Solvent Paramagnetic Relaxation Enhancements. J. Am. Chem. Soc. 136 (29), 10277-10286.

(31) Lan, J., Ge, J., Yu, J., Shan, S., Zhou, H., Fan, S., Zhang, Q., Shi, X., Wang, Q., Zhang, L., and Wang, X. (2020) Structure of the SARSCoV-2 Spike Receptor-Binding Domain Bound to the ACE2 Receptor. Nature 581 (7807), 215-220.

(32) Vorbrodt, A. W. (1987) Demonstration of Anionic Sites on the Luminal and Abluminal Fronts of Endothelial Cells with Poly-LLysine-Gold Complex. J. Histochem. Cytochem. 35 (11), 1261-1266.

(33) Amin, M., Sorour, M. K., and Kasry, A. (2020) Comparing the Binding Interactions in the Receptor Binding Domains of SARS-CoV2 and SARS-CoV. J. Phys. Chem. Lett. 11, 4897-4900.

(34) Wang, Y., Liu, M., and Gao, J. (2020) Enhanced Receptor Binding of SARS-CoV-2 through Networks of Hydrogen-Bonding and Hydrophobic Interactions. Proc. Natl. Acad. Sci. U. S. A. 117 (25), 13967-13974.

(35) Calligari, P., Bobone, S., Ricci, G., and Bocedi, A. (2020) Molecular Investigation of SARS-CoV-2 Proteins and Their Interactions with Antiviral Drugs. Viruses 12 (4), 445.

(36) Sancini, G., Dal Magro, R., Ornaghi, F., Balducci, C., Forloni, G., Gobbi, M., Salmona, M., and Re, F. (2016) Pulmonary Administration of Functionalized Nanoparticles Significantly Reduces Beta-Amyloid in the Brain of an Alzheimer's Disease Murine Model. Nano Res. 9 (7), 2190-2201.

(37) Steardo, L., Steardo, L., Zorec, R., and Verkhratsky, A. (2020) Neuroinfection May Contribute to Pathophysiology and Clinical Manifestations of COVID-19. Acta Physiol. 229, e13473. 
(38) Dijkman, R., Jebbink, M. F., Deijs, M., Milewska, A., Pyrc, K., Buelow, E., van der Bijl, A., and van der Hoek, L. (2012) ReplicationDependent Downregulation of Cellular Angiotensin-Converting Enzyme 2 Protein Expression by Human Coronavirus NL63. J. Gen. Virol. 93 (9), 1924-1929.

(39) Kuba, K., Imai, Y., Rao, S., Gao, H., Guo, F., Guan, B., Huan, Y., Yang, P., Zhang, Y., Deng, W., Bao, L., Zhang, B., Liu, G., Wang, Z., Chappell, M., Liu, Y., Zheng, D., Leibbrandt, A., Wada, T., Slutsky, A. S., Liu, D., Qin, C., Jiang, C., and Penninger, J. M. (2005) A Crucial Role of Angiotensin Converting Enzyme 2 (ACE2) in SARS Coronavirus-Induced Lung Injury. Nat. Med. 11 (8), 875-879.

(40) Watkins, J. (2020) Preventing a Covid-19 Pandemic. BMJ. 368, No. $\mathrm{m} 810$.

(41) Glowacka, I., Bertram, S., Herzog, P., Pfefferle, S., Steffen, I., Muench, M. O., Simmons, G., Hofmann, H., Kuri, T., Weber, F., Eichler, J., Drosten, C., and Pöhlmann, S. (2010) Differential Downregulation of ACE2 by the Spike Proteins of Severe Acute Respiratory Syndrome Coronavirus and Human Coronavirus NL63. J. Virol. 84 (2), 1198-1205.

(42) Terrence, C. F., Rao, G. R., and Perper, J. A. (1981) Neurogenic Pulmonary Edema in Unexpected, Unexplained Death of Epileptic Patients. Ann. Neurol. 9 (5), 458-464.

(43) Šedý, J., Kuneš, J., and Zicha, J. (2015) Pathogenetic Mechanisms of Neurogenic Pulmonary Edema. J. Neurotrauma 32 (15), 1135-1145.

(44) Karimi, N., Sharifi Razavi, A., and Rouhani, N. (2020) Frequent Convulsive Seizures in an Adult Patient with COVID-19: A Case Report. Iran. Red Crescent Med. J. 22, e102828.

(45) Libbey, J. E., and Fujinami, R. S. (2011) Neurotropic Viral Infections Leading to Epilepsy: Focus on Theiler's Murine Encephalomyelitis Virus. Future Virol. 6 (11), 1339-1350.

(46) Huang, C., Wang, Y., Li, X., Ren, L., Zhao, J., Hu, Y., Zhang, L., Fan, G., Xu, J., Gu, X., Cheng, Z., Yu, T., Xia, J., Wei, Y., Wu, W., Xie, X., Yin, W., Li, H., Liu, M., Xiao, Y., Gao, H., Guo, L., Xie, J., Wang, G., Jiang, R., Gao, Z., Jin, Q., Wang, J., and Cao, B. (2020) Clinical Features of Patients Infected with 2019 Novel Coronavirus in Wuhan, China. Lancet 395 (10223), 497-506.

(47) Zhao, M. (2020) Cytokine Storm and Immunomodulatory Therapy in COVID-19: Role of Chloroquine and Anti-IL-6 Monoclonal Antibodies. Int. J. Antimicrob. Agents 55, 105982.

(48) Singhi, P. (2011) Infectious Causes of Seizures and Epilepsy in the Developing World. Dev. Med. Child Neurol. 53 (7), 600-609.

(49) Libbey, J. E., Kennett, N. J., Wilcox, K. S., White, H. S., and Fujinami, R. S. (2011) Interleukin-6, Produced by Resident Cells of the Central Nervous System and Infiltrating Cells, Contributes to the Development of Seizures Following Viral Infection. J. Virol. 85 (14), 6913-6922.

(50) Libbey, J. E., Kirkman, N. J., Smith, M. C. P., Tanaka, T., Wilcox, K. S., White, H. S., and Fujinami, R. S. (2008) Seizures Following Picornavirus Infection. Epilepsia 49 (6), 1066-1074.

(51) Ye, M., Ren, Y., and Lv, T. (2020) Encephalitis as a Clinical Manifestation of COVID-19. Brain, Behav., Immun., DOI: 10.1016/ j.bbi.2020.04.017.

(52) Hummel, T., Whitcroft, K. L., Andrews, P., Altundag, A., Cinghi, C., Costanzo, R. M., Damm, M., Frasnelli, J., Gudziol, H., Gupta, N., Haehner, A., Holbrook, E., Hong, S. C., Hornung, D., Hüttenbrink, K. B., Kamel, R., Kobayashi, M., Konstantinidis, I., Landis, B. N., Leopold, D. A., Macchi, A., Miwa, T., Moesges, R., Mullol, J., Mueller, C. A., Ottaviano, G., Passali, G. C., Philpott, C., Pinto, J. M., Ramakrishnan, V. J., Rombaux, P., Roth, Y., Schlosser, R. A., Shu, B., Soler, G., Stjärne, P., Stuck, B. A., Vodicka, J., and WelgeLuessen, A. (2017) Position Paper on Olfactory Dysfunction. Rhinology 56, 1-30.

(53) Welge-Lüssen, A. (2005) Re-Establishment of Olfactory and Taste Functions. GMS Curr. Top. Otorhinolaryngol. Head Neck Surg. 4, Doc06.

(54) Giacomelli, A., Pezzati, L., Conti, F., Bernacchia, D., Siano, M., Oreni, L., Rusconi, S., Gervasoni, C., Ridolfo, A. L., Rizzardini, G., Antinori, S., and Galli, M. (2020) Self-Reported Olfactory and Taste
Disorders in SARS-CoV-2 Patients: A Cross-Sectional Study. Clin. Infect. Dis., ciaa330.

(55) Bagheri, S. H. R., Asghari, A. M., Farhadi, M., Shamshiri, A. R., Kabir, A., Kamrava, S. K., Jalessi, M., Mohebbi, A., Alizadeh, R. Honarmand, A. A., Ghalehbaghi, B., and Salimi, A. (2020) Coincidence of COVID-19 Epidemic and Olfactory Dysfunction Outbreak. medRxiv, March 27, 2020, ver. 1, DOI: 10.1101/ 2020.03.23.20041889.

(56) Lechien, J. R., Chiesa-Estomba, C. M., De Siati, D. R., Horoi, M., Le Bon, S. D., Rodriguez, A., Dequanter, D., Blecic, S., El Afia, F., Distinguin, L., Chekkoury-Idrissi, Y., Hans, S., Delgado, I. L., CalvoHenriquez, C., Lavigne, P., Falanga, C., Barillari, M. R., Cammaroto, G., Khalife, M., Leich, P., Souchay, C., Rossi, C., Journe, F., Hsieh, J., Edjlali, M., Carlier, R., Ris, L., Lovato, A., De Filippis, C., Coppee, F., Fakhry, N., Ayad, T., and Saussez, S. (2020) Olfactory and Gustatory Dysfunctions as a Clinical Presentation of Mild-to-Moderate Forms of the Coronavirus Disease (COVID-19): A Multicenter European Study. Eur. Arch. Otorhinolaryngol. 277, 2251.

(57) Perlman, S., Evans, G., and Afifi, A. (1990) Effect of Olfactory Bulb Ablation on Spread of a Neurotropic Coronavirus into the Mouse Brain. J. Exp. Med. 172 (4), 1127-1132.

(58) Mohammed, A. K., Magnusson, O., Maehlen, J., Fonnum, F., Norrby, E., Schultzberg, M., and Kristensson, K. (1990) Behavioural Deficits and Serotonin Depletion in Adult Rats after Transient Infant Nasal Viral Infection. Neuroscience 35 (2), 355-363.

(59) Seiden, A. M. (2004) Postviral Olfactory Loss. Otolaryngol. Clin. North Am. 37 (6), 1159-1166.

(60) Dalton, P. (2004) Olfaction and Anosmia in Rhinosinusitis. Curr. Allergy Asthma Rep. 4 (3), 230-236.

(61) Hummel, T., Landis, B. N., and Hüttenbrink, K.-B. (2012) Smell and Taste Disorders. GMS Curr. Top. Otorhinolaryngol. Head Neck Surg. 10, Doc04.

(62) Altschul, S. F., Gish, W., Miller, W., Myers, E. W., and Lipman, D. J. (1990) Basic Local Alignment Search Tool. J. Mol. Biol. 215 (3), 403-410.

(63) Henikoff, S., and Henikoff, J. G. (1992) Amino Acid Substitution Matrices from Protein Blocks. Proc. Natl. Acad. Sci. U. S. A. 89 (22), 10915-10919.

(64) Abagyan, R. A., and Batalov, S. (1997) Do Aligned Sequences Share the Same Fold? J. Mol. Biol. 273 (1), 355-368.

(65) Molsoft LLC. ICM-Browser, Molsoft LLC, http://www.molsoft. com/icm browser.html (accessed Jul 1, 2020-07-01).

(66) RStudio Team (2015) RStudio: Integrated Development for $R$, RStudio, PBC, Boston, MA.

(67) Fiser, A., and Sali, A. (2003) MODELLER: Generation and Refinement of Homology-Based Protein Structure Models. Methods Enzymol. 374, 461-491.

(68) RCSB PDB, https://www.rcsb.org/ (accessed 2020-04-07).

(69) Pettersen, E. F., Goddard, T. D., Huang, C. C., Couch, G. S., Greenblatt, D. M., Meng, E. C., and Ferrin, T. E. (2004) UCSF Chimera - A Visualization System for Exploratory Research and Analysis. J. Comput. Chem. 25 (13), 1605-1612.

(70) Baker, N. A., Sept, D., Joseph, S., Holst, M. J., and McCammon, J. A. (2001) Electrostatics of Nanosystems: Application to Microtubules and the Ribosome. Proc. Natl. Acad. Sci. U. S. A. 98 (18), 10037-10041.

(71) Delano, W. L. The PyMOL Molecular Graphics System. 2002.

(72) Lerner, M. G., and Carlson, H. A. (2006) APBS Plugin for PyMOL, University of Michigan, Ann Arbor.

(73) Massova, I., and Kollman, P. A. (2000) Combined Molecular Mechanical and Continuum Solvent Approach (MM- PBSA/GBSA) to Predict Ligand Binding. Perspect. Drug Discovery Des. 18 (i), 113135.

(74) Kumari, R., Kumar, R, and Lynn, A. (2014) G-Mmpbsa -A GROMACS Tool for High-Throughput MM-PBSA Calculations. J. Chem. Inf. Model. 54 (7), 1951-1962.

(75) Ramos, R. M., and Moreira, I. S. (2013) Computational Alanine Scanning Mutagenesis-An Improved Methodological Ap- 
proach for Protein-DNA Complexes. J. Chem. Theory Comput. 9 (9),

4243-4256.

(76) Berendsen, H. J. C., van der Spoel, D., and van Drunen, R. (1995) GROMACS: A Message-Passing Parallel Molecular Dynamics Implementation. Comput. Phys. Commun. 91 (1-3), 43-56. 